caused by masturbation from $3.9 \%$ (95\% CI 2.0 to 6.8 ) to $7.8 \%(95 \% \mathrm{CI} 4.3$ to 15.6$)$ which was primarily due to solo masturbation (estimates of $3.5 \%$ (95\% CI 1.7 to 6.1 ) to $7.1 \%$ (95\%CI 4.0 to 13.1)) with little contribution from partnered masturbation (estimates of $0.3 \%(95 \% \mathrm{CI} 0.0$ to 1.5$)$ to $0.7 \%$ (95\% CI 0.1 to 4.0$)$ ).

Conclusions Our model suggests that saliva use as a lubricant for solo/partnered masturbation plays a negligible a role in chlamydia transmission in MSM.

\section{STI treatment}

\subsection{TOLERABILITY OF TOPICAL IMIQUIMOD AGAINST HIGH- RISK HUMAN PAPILLOMAVIRUS INFECTION IN MEN- WHO-HAVE-SEX-WITH-MEN LIVING WITH HIV}

\begin{abstract}
1,2 D Durukan*, $1,2 \mathrm{~T}$ Phillips, ${ }^{3,4} \mathrm{G}$ Murray, ${ }^{1,2} \mathrm{~J}$ Ong, ${ }^{5} \mathrm{~A}$ Grulich, ${ }^{5} \mathrm{M}$ Poynten, ${ }^{5} \mathrm{~F}$ Jin, ${ }^{1,2} \mathrm{C}$ Bradshaw, ${ }^{2}$ I Aguirre, ${ }^{2} \mathrm{~J}$ Silvers, ${ }^{2} \mathrm{H}$ Kent,,${ }^{3,4} \mathrm{~S}$ Atchison, ${ }^{4} \mathrm{P}$ Balgovind, ${ }^{3,4,6} \mathrm{~A}$ Cornall, ${ }^{1,2} \mathrm{M}$ Chen, ${ }^{1,2,7} \mathrm{E}$ Chow, ${ }^{1,2} \mathrm{C}$ Fairley. ${ }^{1}$ Central Clinical School, Monash University, Melbourne, Australia; ${ }^{2}$ Melbourne Sexual Health Centre, Alfred Health, Carlton, Australia; ${ }^{3}$ Murdoch Children's Research Institute, Parkville, Australia; ${ }^{4}$ Centre for Women's Infectious Diseases, The Royal Women's Hospital, Parkville; ${ }^{5}$ The Kirby Institute, UNSW, Sydney, Australia; ${ }^{6}$ Department of Obstetrics and Gynaecology, University of Melbourne, Parkville, Australia; ${ }^{7}$ Melbourne School of Population and Global Health, The University of Melbourne, Parkville, Australia
\end{abstract}

10.1136/sextrans-2021-sti.110

Background Men-who-have-sex-with-men (MSM) living with HIV have a high prevalence of high-risk human papillomavirus (hrHPV) in the anus, and a high incidence of anal cancer. We conducted an open-label, single-arm pilot study to assess the utility of imiquimod cream against hrHPV among MSM living with HIV.[ACTRN12617001355369]

Methods The study was conducted at Melbourne Sexual Health Centre between April 2018 and June 2020. MSM aged $\geq 18$ years, living with HIV, who tested positive for any anal hrHPV on clinician-collected swabs were eligible. We instructed men to apply 5\% imiquimod cream $(6.25 \mathrm{mg})$ intra-anally and peri-anally 3 doses per-week for 16-weeks (Phase 1), followed by a maintenance period of 1 dose perweek for 48-weeks (Phase 2). We collected adverse events (AE) using text messages and questionnaires.

Results Thirty MSM were enrolled to phase 1 and 27 completed the week 16 follow-up(median age 50). Twenty-four MSM (86\%) applied at least $50 \%$ of imiquimod doses. All men reported AE, with $39.5 \%$ reporting grade $1,39.5 \%$ grade 2, and 21\% grade 3 AEs. Eighteen MSM (67\%) required treatment interruption. The reasons for interruption were haemorrhoids $(n=3)$, herpes genitalis reactivation $(n=2)$, and grade 2-3 AEs $(n=11)$ such as irritation, itching and tenderness. $60 \%$ of MSM with grade $2(n=6)$ and $33 \%$ with grade 3 AEs $(n=2)$ opted to continue to phase 2. Eighteen MSM were enrolled to phase 2 and 13 completed the week 48 follow-up. All MSM took at least $50 \%$ of doses. No treatmentlimiting AEs were reported. $10 \mathrm{MSM}$ in phase 1 (37\%) and none in phase 2 reported their sex life was negatively impacted from imiquimod use.

Conclusion Intra-anal and peri-anal imiquimod at 3 doses perweek was poorly tolerated over 16 weeks and most men required treatment interruption due to AEs. In contrast, oncea-week application was well tolerated with no treatment-limiting AEs reported over 48-weeks.

\subsection{TREATMENT EFFICACY OF 1G AZITHROMYCIN VERSUS 100MG DOXYCYCLINE BI-DAILY FOR SEVEN DAYS FOR ASYMPTOMATIC RECTAL CHLAMYDIA TRACHOMATIS}

${ }^{1} \mathrm{~A}$ Lau* ${ }^{*}{ }^{1} \mathrm{~F}$ Kong, ${ }^{2,3} \mathrm{C}$ Fairley, ${ }^{4} \mathrm{D}$ Templeton, ${ }^{5} \mathrm{Amin},{ }^{1} \mathrm{~S}$ Phillips, ${ }^{6} \mathrm{M}$ Law, ${ }^{2,3} \mathrm{M}$ Chen, ${ }^{2,3} \mathrm{C}$ Bradshaw, ${ }^{6} \mathrm{~B}$ Donovan, ${ }^{7} \mathrm{~A}$ McNulty, ${ }^{8} \mathrm{M}$ Boyd, ${ }^{9} \mathrm{P}$ Timms, ${ }^{1,2,3} \mathrm{E}$ Chow, ${ }^{6} \mathrm{D}$ Regan, ${ }^{10} \mathrm{C}$ Khaw, ${ }^{11} \mathrm{D}$ Lewis, ${ }^{6} \mathrm{~J}$ Kaldor, ${ }^{10} \mathrm{M}$ Ratnayake, ${ }^{1} \mathrm{~N}$ Carvalho, ${ }^{1 \mathrm{~J}}$ Hocking. ${ }^{1}$ Melbourne School of Population and Global Health, The University of Melbourne, Carlton, Australia; ${ }^{2}$ Melbourne Sexual Health Centre, Alfred Health, Carlton, Australia; ${ }^{3}$ Central Clinical School, Monash University, Carlton, Australia; ${ }^{4}$ RPA Sexual Health, Camperdown, Australia; ${ }^{5}$ Macquarie University, Macquarie Park, Australia; ${ }^{6}$ The Kirby Institute, Kensington, Australia; ${ }^{7}$ Sydney Sexual Health Centre, Sydney, Australia; ${ }^{8}$ Adelaide Medical School, University of Adelaide, Adelaide, Australia; ${ }^{9}$ Genecology Research Centre, University of the Sunshine Coast, Sippy Downs, Australia; ${ }^{10}$ Adelaide Sexual Health Centre, Adelaide, Australia; ${ }^{11}$ Western Sydney Sexual Health Centre, Parramatta, Australia

10.1136/sextrans-2021-sti.111

Background Rectal chlamydia is the most commonly diagnosed bacterial sexually transmissible infection among men who have sex with men (MSM) and there is increasing concern about rectal chlamydia in women.

The absence of randomised controlled trial (RCT) evidence means there is ongoing debate about the most efficacious treatment. Methods We conducted a double-blind double-dummy RCT to compare the efficacy of azithromycin $1 \mathrm{~g}$ singledose with 7 days doxycycline $100 \mathrm{mg}$ twice daily for the treatment of asymptomatic rectal chlamydia.

MSM diagnosed with asymptomatic rectal chlamydia infection at five sexual health clinics in Australia were randomly assigned to receive either doxycycline or azithromycin. Primary outcome was rectal chlamydia microbiological cure defined as a negative nucleic acid amplification test at four weeks. Logistic regression was used to calculate the difference in microbial cure within a modified intention to treat population that excluded L2 serovar diagnoses post-recruitment.

Results We enrolled 625 men and randomly assigned 314 to doxycycline and 311 to azithromycin between August 2016 and August 2019. Primary outcome data were available for 290 (92\%) assigned to doxycycline and 297 (96\%) assigned to azithromycin. In the modified intention to treat population, the observed microbiological cure was 281/290 (96.9\%; 95\% CI: 94.9, 98.9) for doxycycline and 227/297 (76.4\%; 95\%CI: $73.8,79.1)$ for azithromycin, with an adjusted risk difference of $19.9 \%$ (95\% CI: 14.6, 25.3; p<0.001) in favour of doxycycline. Adverse events including nausea, diarrhoea and vomiting were reported by $33.8 \%$ (98/290) receiving doxycycline and $45.1 \%(134 / 297)$ azithromycin (risk difference=-11.3\%; 95\%CI: -19.5, -3.2). Chlamydial load at baseline was greater for those in the azithromycin arm who failed treatment compared to those who did not.

Conclusions The efficacy of doxycycline was found to be substantially superior to azithromycin in the treatment of asymptomatic rectal chlamydia infection among MSM. Doxycycline must replace azithromycin as firstline treatment for symptomatic rectal chlamydia.

\subsection{CLINICAL IMPROVEMENT AFTER TREATMENT FOR URETHRITIS: THE ROLE OF MYCOPLASMA GENITALIUM}

1J Braam* ${ }^{*}$ 1,2 A van Dam, ${ }^{1,2} \mathrm{~S}$ Bruisten, ${ }^{1} \mathrm{M}$ van Rooijen, ${ }^{1,2} \mathrm{H}$ de Vries, ${ }^{1,2} \mathrm{M}$ Schim van der Loeff, ${ }^{1,3} \mathrm{C}$ Vergunst. ${ }^{1}$ Public Health Service of Amsterdam, Amsterdam, The Netherlands; ${ }^{2}$ Amsterdam University Medical Center (UMC), Amsterdam, The Netherlands; ${ }^{3}$ NWZ, Den Helder, The Netherlands

10.1136/sextrans-2021-sti. 112 